\title{
MATERNAL SMOKING DURING PREGNANCY AND BIRTH OUTCOMES IN A SAMPLE OF ROMANIAN WOMEN
}

\author{
Cristian I. Meghea ${ }^{1,2}$, loana A. Rus ${ }^{2}$, Răzvan M. Cherecheş ${ }^{2}$, Nicolae Costin ${ }^{3}$, Gabriela Caracostea ${ }^{4}$, Alexandra \\ Brînzaniuc ${ }^{2}$ \\ ${ }^{1}$ Institute for Health Policy and Department of Obstetrics, Gynecology and Reproductive Biology, College of Human Medicine, Michigan State \\ University, East Lansing, Michigan, USA \\ ${ }^{2}$ Centre for Health Policy and Public Health, Institute for Social Research, Faculty of Political, Administrative and Communication Science, \\ Babes-Bolyai University, Cluj-Napoca, Romania \\ ${ }^{3}$ Emergency Hospital Octavian Fodor, Obstetrics and Gynecology Section II, Cluj-Napoca, Romania \\ ${ }^{4}$ Emergency Hospital Octavian Fodor, Obstetrics and Gynecology Section I, Cluj-Napoca, Romania
}

\section{SUMMARY}

Smoking during pregnancy is causally associated with reduced birth weight and is strongly related to preterm birth. Smoking cessation in early pregnancy seems to reduce these risks, although the research evidence is limited. In a sample of Romanian women, differences in birth outcomes were assessed between non-smokers and women who continued to smoke during pregnancy and non-smokers and women who stopped smoking when they found out about the pregnancy. Pregnant women were recruited in two urban clinics $(\mathrm{N}=474)$. A baseline questionnaire collected information on their smoking status, depressive symptoms, stress, demographics, and other characteristics at recruitment. The women reported the newborn weight and birth term by phone in the first weeks following birth. Descriptive statistics and multivariate regressions were used to analyze the relationship between smoking status during pregnancy and birth outcomes. Over $61 \%(N=290)$ women were non-smokers, $15 \%(N=72)$ smoked during pregnancy, and $24 \%(\mathrm{~N}=112)$ quit smoking when they found out about the pregnancy. Compared to non-smokers, continuous smokers delivered babies 165 grams lighter $(95 \% \mathrm{Cl}-313,-17)$. Women who stopped smoking when they ascertained the pregnancy had higher odds of delivering a newborn who was small for gestational age compared to non-smokers $(\mathrm{OR}=2.16,95 \% \mathrm{Cl} 1.05,4.43)$. Elevated maternal stress was associated with reduced birth weight $(-113$ grams, $95 \% \mathrm{Cl}-213,-11)$, and higher odds of a preterm birth $(\mathrm{OR}=2.8,95 \% \mathrm{Cl} 1.17$, 6.76). In a predominantly urban sample of Romanian women, continuous maternal smoking during pregnancy was a risk factor for restricted foetal growth. Smoking cessation when the pregnancy was ascertained did not seem to reduce this risk. Smoking prevention efforts should therefore begin before pregnancy and should integrate psychological components, addressing maternal stress in particular.

Key words: pregnancy, smoking, birth outcomes, preterm, small for gestational age, Romania

Address for correspondence: C. I. Meghea, Michigan State University, 965 Fee Rd, Room A632-B, East Lansing, MI 48823, USA. E-mail: Cristian.Meghea@hc.msu.edu

\section{INTRODUCTION}

Smoking during pregnancy is causally associated with reduced birth weight, and existing evidence suggests that smoking is strongly related to preterm birth, placental abruption, stillbirth, ectopic pregnancies, and placenta previa (1). Review studies have established the causal effects of pregnancy smoking on foetal growth restriction, and increasing evidence suggests that pregnancy smoking may cause preterm birth, although the reported smoking-related risks of preterm birth are relatively modest in size (1-3). Many of the performed studies were not able to distinguish between continuous smokers during pregnancy and those who quit during pregnancy. Those who did, however, found that smoking cessation improves foetal growth, and increases gestational periods (1-5). The few studies of women who stopped smoking early in the pregnancy showed that the birth weight, delivery gestational age, and the risks of preterm and small for gestational age births were the same as those of infants born to non-smokers
(6-8). In addition, other factors not always accounted for in prior studies, may have confounded the relationship between prenatal smoking and birth outcomes, in particular stress and mental health during pregnancy $(9,10)$. Most of the available research on the relationship between prenatal smoking and birth outcomes has used data from developed countries. The majority of the studies included in systematic reviews focused on populations in Western Europe and North America (1-3). The evidence from Central and Eastern Europe (CEE) and other developing regions is limited.

Smoking prevalence has increased through the 1990s in the former communist countries in CEE, especially among young women $(1,11)$. In Romania, there was a significant increase in smoking among women from $11 \%$ smoking in 1989 to $30 \%$ in 1999. A recent national survey indicated that smoking among women has decreased to $16.7 \%$ in Romania, with the highest smoking rate in the $25-44$ age group at $24 \%$ (12). A recent study on tobacco use in 3 billion individuals from 16 countries, including 14 low and middle-income countries, the UK, and the 
US, reported smoking rates among women ranging from $0.5 \%$ in Egypt and below 3\% in China and India, to 11\% in Ukraine, $15 \%$ in Turkey, and over $24 \%$ in Poland (13). The smoking rate among women in the US was $17.5 \%$ (14).

The research literature on smoking during pregnancy in the CEE region is not voluminous, nevertheless, it has been increasing in the recent years. We previously found a self-reported smoking rate during pregnancy of $15 \%$, with an additional $24 \%$ women reporting quitting when they ascertained the pregnancy in a large urban sample in Romania (11). A few other CEE studies found self-reported smoking rates of $24 \%$ ever during pregnancy in a large-sample Romanian study (15) and 26\% in the third pregnancy trimester in Serbia (16). A Polish study found 25-30\% selfreported and $33-41 \%$ biochemically verified smoking rate during pregnancy (17). Smoking during pregnancy was substantially lower in many developed countries, with rates between 9-15\% in the USA, Canada and Europe (1, 18-20).

We proposed an assessment of the impact of smoking during pregnancy on birth outcomes in a sample of Romanian women. Our contributions are the differentiation between continuous smokers and women who quit when they found out about their pregnancy, in an unexplored population, living in different social, economic, and cultural environment than previously studied populations; dose-response analyses of the effects of pregnancy smoking and cessation on birth outcomes; and adjustments for potential confounding factors, some previously undocumented in the region. The specific objectives of this study were to assess differences in birth outcomes - small for gestational age (SGA) birth, preterm birth, birth weight, and gestational age at birth between non-smokers and women who continued to smoke during pregnancy; and non-smokers and women who stopped smoking when they learnt about the pregnancy. This provides additional evidence for approaching smoking cessation before and during pregnancy in the context of improving maternal and child health, facilitating potential efforts in Romania in particular.

\section{MATERIALS AND METHODS}

This study is based on the Smoking during Pregnancy in Romania - SPRO project $(11,21)$. Briefly, pregnant women aged 18 or older who sought antenatal care or were confined in bed in the two largest obstetrics-gynecology clinics in Cluj-Napoca, Romania, between November 2008 and August 2009 were invited to participate in the SPRO project. The response rate at recruitment during pregnancy was over $80 \%$ at both clinics. The total number of recruited pregnant women was 916 (11). Baseline data at recruitment was self-reported through a detailed questionnaire (21). The present study is based on 474 women who were followed-up by phone in the first weeks after birth (follow-up rate 52\%). The study was approved by the Institutional Review Board of BabesBolyai University in Cluj-Napoca, Romania. Details about the SPRO project were described elsewhere (11). Informed consent was obtained from each participant.

\section{Smoking during Pregnancy}

Cigarette smoking status was self-reported (not smoking, quitting upon learning about pregnancy, and continuous smoking during pregnancy), and was defined relying on the multiple choice question 'Do you currently smoke cigarettes?' The question was part of the baseline interview at recruitment during pregnancy. The first answer option was 'Yes', the second 'I do not smoke now and did not before pregnancy', and two options for quitting, which we grouped together: 'I quit since learning I was pregnant and I intend to stay smoke free' and 'I quit since learning I was pregnant and I will probably smoke again after delivery'. The question was initially developed by Mullen et al. (22) and was previously used in the research literature $(23,24)$. We adapted it to measure postnatal relapse intentions of self-reported quitters.

\section{Outcomes}

Birth weight (in grams) and completed gestation weeks at birth were self-reported during the follow-up phone interview in the first weeks after delivery. Small for gestational age (SGA) was defined as birth weight below the 10th percentile. We generated growth charts for our study population using the Gestation Related Average Weight (GRAW) tool (www.gestation.net/fetal_growth/ graw/index.htm). Gestational age at birth was expressed in completed pregnancy weeks. Preterm births were defined as deliveries with a gestational age at birth less than 37 completed weeks.

\section{Covariates}

The covariates were described in detail elsewhere (11). Briefly, the Romanian translation of the Patient Health Questionnaire-2 (PHQ-2) screening tool (25) was used to binary assess depressive symptoms during pregnancy, and a high stress binary indicator was defined based on a stress score equal or higher than the median sample value of 4 measured on the Perceived Stress Scale-4 (26). Other binary covariates included age 35 or older; education college or higher; rural living; unmarried; an indicator for unwanted pregnancy; and an indicator of whether the pregnancy was the first. Over $68 \%$ of the women in our sample were interviewed at baseline during one of their prenatal visits. The other $32 \%$ were hospitalized in the clinics at the baseline interview, as recommended by their prenatal provider, because of a difficult pregnancy. A binary indicator distinguished between these two categories.

\section{Statistical Analysis}

We analyzed reported descriptive statistics (counts and percentages in Table 1) to explore unadjusted differences in birth outcomes and various characteristics between non-smokers, women who quit when they learnt about the pregnancy, and continuous smokers during pregnancy. We used chi-square or Fisher's exact test to compare categorical variables, as appropriate, and Student's t test for comparing continuous variables between the three smoking groups. We used multiple regressions (linear ordinary least squares regression, logistic regression) to identify the effects of non-smoking and quitting, adjusting for other potential confounders. Twin births were excluded from the multivariate analyses $(n=9)$.

To account for potential differences between the pregnant women confined to bed and the rest of the sample, we included a 'confined' indicator for women who were hospitalized at the 
baseline interview. As approximately four percent of the 'confined' indicator and stress scores were missing, we recoded the missing values to 'no' and added 'missing - yes/no' indicators in the regression analyses in order to maximize the number of observations retained in the analyses.

As the baseline data were collected in two prenatal hospitals and the vast majority gave birth in the same two hospitals, we tested for nesting within locations. We performed multiple regressions accounting for (xtmixed and xtmelogit in Stata), and then ignoring, the hierarchical structure of the data. Likelihood ratio tests found no evidence of nesting within the hospitals. We reported non-nested regression results (Tables 2 and 3). All analyses were performed using Stata (Stata Statistical Software, release 11; Stata Corp, College Station, TX, USA).

\section{RESULTS}

\section{Baseline Characteristics and Bivariate Analyses}

Except for a higher percentage of college graduates $(p=0.03)$ and a lower share of women who were confined in bed at the time of the baseline interview $(p=0.08)$, there were no other statistically significant differences between women who answered the follow-up questions after birth - the subjects of this study - and those who were lost to follow-up (unreported results, available from the authors).

Table 1 indicates that smokers had a higher prevalence of elevated stress during pregnancy $(61.9 \%)$ compared to non-smokers $(50.1 \%)$ and women who quit when they found out about the pregnancy $(51.9 \%)$. Smokers were less likely to be 35 or older and to have graduated college $(5.7 \%$ and $21.1 \%$ ) compared to non-smokers (12.6\% and $62.4 \%)$ and those who quit (14.5\% and 50.9\%).

The average birth weight in our sample was 3,340 grams. Infants born by women who smoked during pregnancy had the lowest birth weight $(3,176$ grams on average) compared to women who quit smoking (3,340 grams) and non-smokers (3,382 grams). Smokers had higher rates of SGA $(15.7 \%)$ and preterm birth $(11.3 \%)$ compared to non-smokers $(7.4 \%$ and $6.6 \%)$, and women who quit during pregnancy (15.6\% and 9.8\%).

\section{Multiple Regression Analyses}

Tables 2 and 3 report the results of multiple regression analyses for four birth outcomes: birth weight (in grams), gestational age at birth (weeks), SGA, and preterm birth. Compared to women who did not smoke during pregnancy, smokers delivered, on average, babies 165 grams lighter (95\% CI -313, -17). Smokers also delivered at shorter gestational ages $(-0.25$ weeks $)$ and had higher odds of SGA $(\mathrm{OR}=1.79)$ and preterm birth $(\mathrm{OR}=1.29)$, but the results were not statistically significant. Unreported results indicated that, compared to women who did not smoke during pregnancy, the babies whose mothers smoked 10 or more cigarettes daily were $248 \mathrm{~g}$ lighter $(95 \% \mathrm{CI}-491,-5)$, while the babies whose mothers smoked fewer than 10 cigarettes daily were $135 \mathrm{~g}$ lighter $(95 \% \mathrm{CI}-301,-32)$. Further, the SGA odds ratio for women who smoked 10 or more cigarettes daily was $2.01 \mathrm{com}-$ pared to non-smokers, while the SGA odds ratio for women who smoked fewer than 10 cigarettes daily was 1.34 , but the results were not statistically significant.

Women who stopped smoking when they ascertained their pregnancy had higher odds of SGA $(\mathrm{OR}=2.16,95 \% \mathrm{CI} 1.05,4.43)$ compared to non-smokers (Table 2). Women who stopped smoking also delivered lighter babies $(-48 \mathrm{~g})$, at shorter gestational ages $(-0.16$ weeks $)$, and had higher odds of preterm birth $(\mathrm{OR}=1.41)$, but the results were not statistically significant (Tables 2 and 3 ).

Women who reported high levels of stress during pregnancy delivered newborns of smaller weight ( -113 grams, $95 \%$ CI -213 , $-11)$, and had higher odds of preterm birth $(\mathrm{OR}=2.8,95 \% \mathrm{CI}$ $1.2,6.8)$ compared to women reporting low stress. High stress

Table 1. Characteristics of pregnant women followed-up after birth by pregnancy smoking status

\begin{tabular}{|l|c|c|c|c|c|}
\hline \multirow{2}{*}{} & All & Non-smokers & Quitters & Smokers & \multirow{2}{*}{ P value } \\
\cline { 2 - 5 } & $\mathrm{N}=\mathbf{4 7 4}$ & $\mathbf{N = 2 9 0}$ & $\mathbf{N = 1 1 2}$ & $\mathrm{N}=\mathbf{7 2}$ & \\
\hline Depressive symptoms (PHQ-2 positive) & $64.8 \%$ & $65.5 \%$ & $65.8 \%$ & $61.1 \%$ & 0.75 \\
\hline High stress & $52.4 \%$ & $50.1 \%$ & $51.9 \%$ & $61.9 \%$ & 0.28 \\
\hline Age $\geq 35$ years & $12.0 \%$ & $12.6 \%$ & $14.5 \%$ & $5.7 \%$ & 0.09 \\
\hline Education, college or higher & $53.5 \%$ & $62.4 \%$ & $50.9 \%$ & $21.1 \%$ & $<0.01$ \\
\hline Live in rural area & $23.3 \%$ & $20.9 \%$ & $13.6 \%$ & $48.5 \%$ & $<0.01$ \\
\hline Unmarried & $11.5 \%$ & $7.3 \%$ & $11.8 \%$ & $28.2 \%$ & $<0.01$ \\
\hline Unwanted pregnancy & $18.8 \%$ & $18.1 \%$ & $16.5 \%$ & $25.4 \%$ & 0.41 \\
\hline First pregnancy & $59.6 \%$ & $56.1 \%$ & $67.0 \%$ & $62.0 \%$ & 0.19 \\
\hline Confined to bed during pregnancy & $31.6 \%$ & $32.7 \%$ & $29.7 \%$ & $29.9 \%$ & 0.86 \\
\hline Birth weight (grams) & 3,340 & 3,382 & 3,340 & 3,176 & $0.02^{\mathrm{a}}$ \\
\hline Small for gestational age newborn & $10.6 \%$ & $7.4 \%$ & $15.6 \%$ & $15.7 \%$ & 0.02 \\
\hline Gestational age at birth (weeks) & 39.1 & 39.2 & 38.9 & 38.8 & 0.67 \\
\hline Preterm birth (<37 weeks) & $8.0 \%$ & $6.6 \%$ & $9.8 \%$ & $11.3 \%$ & 0.44 \\
\hline
\end{tabular}

Percentages and means are calculated based on the valid, non-missing, responses.

$\mathrm{P}$ values are for comparisons between the three groups using chi-square or analysis of variance tests, with post hoc Tukey test for pairwise comparisons of continuous variables.

${ }^{a}$ Non-smokers vs. smokers $(p<0.05)$. There were no other pairwise differences $(p<0.05)$ in the continuous variables. 
Table 2. Associations between birth weight and term, pregnancy smoking status, mental health, demographics, and other characteristics. Multiple linear regression analysis was used.

\begin{tabular}{|l|c|c|c|c|}
\hline \multirow{2}{*}{} & \multicolumn{2}{|c|}{ Birth weight } & \multicolumn{2}{c|}{ Gestational age at birth } \\
\cline { 2 - 5 } & grams & $95 \% \mathrm{Cl}$ & weeks & $95 \% \mathrm{Cl}$ \\
\hline Smoking (vs. non-smoking) & $-165^{* *}$ & $(-313,-17)$ & -0.25 & $(-0.82,0.32)$ \\
\hline Quitting (vs. non-smoking) & -48 & $(-163,67)$ & -0.16 & $(-0.60,0.29)$ \\
\hline Depressive symptoms (PHQ-2 positive) & -17 & $(-120,86)$ & -0.05 & $(-0.45,0.34)$ \\
\hline High stress & $-113^{* *}$ & $(-213,-11)$ & $-0.38^{*}$ & $(-0.77,0.01)$ \\
\hline Age $\geq 35$ years & $-141^{*}$ & $(-296,13)$ & -0.13 & $(-0.73,0.46)$ \\
\hline Education, college or higher & -7 & $(-112,98)$ & -0.22 & $(-0.62,0.18)$ \\
\hline Live in rural area & -40 & $(-157,77)$ & 0.18 & $(-0.28,0.62)$ \\
\hline Unmarried & -114 & $(-267,37)$ & -0.39 & $(-0.97,0.20)$ \\
\hline Unwanted pregnancy & -73 & $(-193,47)$ & -0.03 & $(-0.50,0.43)$ \\
\hline First pregnancy & $-102^{* *}$ & $(-202,-2)$ & 0.01 & $(-0.39,0.39)$ \\
\hline Confined to bed during pregnancy & 19 & $(-86,123)$ & $-0.69^{* * *}$ & $(-1.63,-0.49)$ \\
\hline
\end{tabular}

${ }^{* \star *},{ }^{* *},{ }^{*}$ denote $p$-values $<0.01 ;<0.05$, and $<0.10$

Table 3. Associations between small for gestational age and preterm birth, pregnancy smoking status, mental health, demographics, and other characteristics. Multiple logistic regression analysis was used.

\begin{tabular}{|l|c|c|c|c|}
\hline \multirow{2}{*}{} & \multicolumn{2}{|c|}{ SGA } & \multicolumn{2}{c|}{ Preterm birth } \\
\cline { 2 - 5 } & Odds Ratio & $95 \% \mathrm{Cl}$ & Odds Ratio & $95 \% \mathrm{Cl}$ \\
\hline Smoking (vs. non-smoking) & 1.79 & $(0.74,4.32)$ & 1.29 & $(0.46,3.67)$ \\
\hline Quitting (vs. non-smoking) & $2.16^{* *}$ & $(1.05,4.43)$ & 1.41 & $(0.59,3.37)$ \\
\hline Depressive symptoms (PHQ-2 positive) & 0.93 & $(0.46,1.84)$ & 0.98 & $(0.43,2.22)$ \\
\hline High stress & 1.47 & $(0.73,2.93)$ & $2.81^{* *}$ & $(1.17,6.76)$ \\
\hline Age $\geq 35$ years & 1.52 & $(0.57,4.07)$ & 0.94 & $(0.29,3.00)$ \\
\hline Education, college or higher & 0.63 & $(0.31,1.28)$ & 1.02 & $(0.44,2.38)$ \\
\hline Live in rural area & 1.47 & $(0.71,3.04)$ & 1.73 & $(0.74,4.04)$ \\
\hline Unmarried & 1.59 & $(0.68,3.73)$ & 0.84 & $(0.26,2.71)$ \\
\hline Unwanted pregnancy & 1.11 & $(0.52,2.40)$ & 1.09 & $(0.44,2.71)$ \\
\hline First pregnancy & 1.26 & $(0.64,2.48)$ & 1.45 & $(0.65,3.21)$ \\
\hline Confined to bed during pregnancy & 0.89 & $(0.45,1.77)$ & $2.41^{* *}$ & $(1.13,5.12)$ \\
\hline
\end{tabular}

SGA: small for gestational age baby; Preterm birth: birth before 37 completed pregnancy weeks.

${ }^{* *}$ denote $\mathrm{p}$-values $<0.05$

was also associated with lower gestational age at birth $(-0.38$ weeks) but the results were only marginally statistically significant $(p=0.06)$. Women aged 35 or above $(-141 \mathrm{~g}, 95 \% \mathrm{CI}-296$, $-13)$ and women who had no prior pregnancies $(-101 \mathrm{~g}, 95 \% \mathrm{CI}$ $-202,-2)$ delivered smaller weight babies.

\section{DISCUSSION}

Continued smoking during pregnancy was significantly associated with lower birth weight compared to not smoking in a sample of Romanian women. There was a dose-response relationship between the number of cigarettes smoked and birth weight. Continued smoking during pregnancy was also associated with higher odds of SGA (including a suggested dose-response relationship), reduced gestational ages at birth, and higher risk of preterm birth, but the estimates were imprecise. Women who quit smoking upon learning about their pregnancy did not have a similar risk of
SGA to non-smokers. High stress during pregnancy was strongly associated with reduced birth weight and higher odds of preterm birth and showed marginally significant associations with lower gestational age at birth.

This was one of the very few studies in CEE linking smoking in pregnancy to birth outcomes. We also contributed to the limited research literature studying the link between quitting smoking early during pregnancy and birth outcomes. Our findings on the link between pregnancy smoking and birth outcomes are consistent with the existing literature investigating populations in Northern America and Western Europe. Prior literature found that smoking during pregnancy was causally associated with reduced birth weight, including a dose-response relationship, while the smokingrelated risk of prematurity was relatively modest $(1-3,27)$. There were few prior studies on the relationship between smoking cessation during pregnancy and birth outcomes. The incidences of low birth weight, SGA, and preterm birth among mothers who quit early, in general, by the end of the first pregnancy trimester 
or early second trimester, were similar to those of non-smokers $(7,8,28)$. Unlike some of the existing literature, our findings suggest that the adverse effects of smoking on foetal growth may not have been reduced by quitting when pregnancy was ascertained.

The current study also found a large and statistically significant association between high perceived stress during pregnancy and adverse birth outcomes, independent from smoking and other risk factors. Women with high levels of perceived stress had children of lower birth weight, and had higher rates of prematurity compared to other women. Prior literature investigating perceived stress and birth outcomes has produced mixed findings. Perceived stress was associated with prematurity in some studies, but usually in combination with other stress measures $(10,29)$. A more recent study (30) found that perceived stress before 20 weeks of gestation was associated with pregnancy complications, especially preterm birth.

We did not biochemically validate smoking status during pregnancy, which was the main limitation of our study. It is possible that some of the women who reported they quit when they found out about the pregnancy in fact continued to smoke $(6,17)$. In addition, women who stopped smoking may not have recognized their pregnancy, and continued to smoke, until later than the first trimester. These could be alternative explanations for our SGA findings. An additional limitation of the study was our inability to assess exposure to environmental tobacco smoke (ETS). It is unclear how this limitation may influence our findings because the evidence on the effects of ETS, in addition to pregnancy smoking, on adverse birth outcomes is mixed (16, 31, 32). A Gestation Related Optimal Weight (GROW) chart was not available for Romania, thus, as an alternative, we calculated SGA using the Gestation Related Average Weight (GRAW) based on the average birthweight at term in our sample (www.gestation. net). However, SGA, accounting for both foetal growth as well as gestational age at delivery, better identifies infants at higher morbidity and mortality risks than low birth weight (birth weight $<2,500 \mathrm{~g}$ ). As this study relied on cross-sectional data, it could not determine causal effects of smoking and quitting during pregnancy on birth outcomes. A final limitation was that our data consisted of a sample of mostly urban women, which was not representative of the Romanian population of women or of the childbearing age female population in Romania.

To conclude, smoking during pregnancy was a risk factor for restricted foetal growth, and quitting when the pregnancy was ascertained did not seem to reduce this risk in a predominantly urban sample of Romanian women. Maternal perceived stress during pregnancy was a risk factor for prematurity and reduced foetal growth. Our findings reinforce the need for smoking prevention efforts targeted at women of childbearing age to begin before a pregnancy. Smoking cessation efforts in Romania should integrate psychological components to address maternal antenatal stress in particular.

\section{Conflict of Interests}

None declared

\section{Funding and adherence to ethical recommendations}

This study was supported, in part, by Unitatea Executiva pentru Finantarea Invatamantului Superior si a Cercetarii Stiintifice Universitare (UEFISCSU) grant number 1/30.06.2008; Bucharest, Romania (to C.I.M.). The content of this manuscript is solely the responsibility of the authors and does not necessarily represent the official views of the funding agency, Michigan State University, or Babes-Bolyai University.

The study was approved by the Institutional Review Board of BabesBolyai University, Centre for Health Policy and Public Health, in ClujNapoca, Romania.

\section{REFERENCES}

1. Cnattingius $\mathrm{S}$. The epidemiology of smoking during pregnancy: smoking prevalence, maternal characteristics, and pregnancy outcomes. Nicotine Tob Res. 2004 Apr;6 Suppl 2:S125-40.

2. Behrman RE, Butler AS, editors. Preterm birth: causes, consequences, and prevention. Washington, DC: National Academies Press; 2007.

3. Savitz DA, Murnane P. Behavioral influences on preterm birth: a review. Epidemiology. 2010 May;21(3):291-9.

4. Li CQ, Windsor RA, Perkins L, Goldenberg RL, Lowe JB. The impact on infant birth weight and gestational age of cotinine-validated smoking reduction during pregnancy. JAMA. 1993 Mar 24-31;269(12):1519-24.

5. Lumley J, Chamberlain C, Dowswell T, Oliver S, Oakley L, Watson L. Interventions for promoting smoking cessation during pregnancy. Cochrane Database Syst Rev. 2009 Jul 8;(3):CD001055.

6. Jaddoe VWV, Troe EJWM, Hofman A, Mackenbach JP, Moll HA, Steegers EAP, et al. Active and passive maternal smoking during pregnancy and the risks of low birthweight and preterm birth: the Generation R Study. Paediatr Perinat Epidemiol. 2008 Mar;22(2):162-71.

7. McCowan LME, Dekker GA, Chan E, Stewart A, Chappell LC, Hunter $\mathrm{M}$, et al.; SCOPE consortium. Spontaneous preterm birth and small for gestational age infants in women who stop smoking early in pregnancy: prospective cohort study. BMJ. 2009 Mar 26;338:b1081. doi: 10.1136/ bmj.b1081.

8. Polakowski LL, Akinbami LJ, Mendola P. Prenatal smoking cessation and the risk of delivering preterm and small-for-gestational-age newborns. Obstet Gynecol. 2009 Aug;114(2 Pt 1):318-25.

9. Goldenberg RL, Culhane JF, Iams JD, Romero R. Epidemiology and causes of preterm birth. Lancet. 2008 Jan 5;371(9606):75-84.

10. Hobel CJ, Goldstein A, Barrett ES. Psychosocial stress and pregnancy outcome. Clin Obstet Gynecol. 2008 Jun;51(2):333-48.

11. Meghea CI, Rus D, Rus IA, Summers Holtrop J, Roman L. Smoking during pregnancy and associated risk factors in a sample of Romanian women. Eur J Public Health. 2012 Apr;22(2):229-33.

12. Irimie S, editor. Global adult tobacco survey - Romania 2011. ClujNapoca: Eikon; 2012.

13. Giovino GA, Mirza SA, Samet JM, Gupta PC, Jarvis MJ, Bhala N, et al.; GATS Collaborative Group. Tobacco use in 3 billion individuals from 16 countries: an analysis of nationally representative cross-sectional household surveys. Lancet. 2012 Aug 18;380(9842):668-79.

14. Schiller JS, Lucas JW, Ward BW, Peregoy JA. Summary health statistics for U.S. adults: National Health Interview Survey, 2010. Vital Health Stat 10. 2012 Jan;(252):1-207.

15. Moga M, Preda GH. Smoking in pregnancy. J Environ Prot Ecol. 2008;9(3):566-73.

16. Krstev S, Marinković J, Simić S, Kocev N, Bondy SJ. Prevalence and predictors of smoking and quitting during pregnancy in Serbia: results of a nationally representative survey. Int J Public Health. 2012 Dec;57(6):87583.

17. Polańska K, Hanke W, Sobala W, Jurewicz J. Exposure to tobacco smoke of pregnant women - results of prospective study in Lodz region. Przegl Lek. 2007;64(10):824-6. (In Polish.)

18. Al-Sahab B, Saqib M, Hauser G, Tamim H. Prevalence of smoking during pregnancy and associated risk factors among Canadian women: a national survey. BMC Pregnancy Childbirth. 2010 May 24;10:24. doi:10.1186/1471-2393-10-24.

19. Tong VT, Dietz PM, Morrow B, D'Angelo DV, Farr SL, Rockhill KM, et al.; Centers for Disease Control and Prevention. Trends in smoking before, during, and after pregnancy - Pregnancy Risk Assessment Monitoring System, United States, 40 sites, 2000-2010. MMWR Surveill Summ. 2013 Nov 8;62(6):1-19.

20. Schneider S, Schütz J. Who smokes during pregnancy? A systematic literature review of population-based surveys conducted in developed countries between 1997 and 2006. Eur J Contracept Reprod Health Care. 2008 Jun;13(2):138-47. 
21. Meghea CI, Rus D, Dirle IA. Characteristics and health behaviors of pregnant women in Romania. Gineco.eu. 2010 Sep;6(3):166-71.

22. Mullen PD, Carbonari JP, Tabak ER, Glenday MC. Improving disclosure of smoking by pregnant women. Am J Obstet Gynecol. 1991 Aug;165(2):409-13.

23. Holtrop JS, Meghea C, Raffo JE, Biery L, Chartkoff SB, Roman L. Smoking among pregnant women with Medicaid insurance: are mental health factors related? Matern Child Health J. 2010 Nov;14(6):971-7.

24. Roman LA, Meghea CI, Raffo JE, Biery HL, Chartkoff SB, Zhu Q, et al. Who participates in state sponsored Medicaid enhanced prenatal services? Matern Child Health J. 2010 Jan;14(1):110-20.

25. Kroenke K, Spitzer RL, Williams JBW. The Patient Health Questionnaire-2: validity of a two-item depression screener. Med Care. 2003 Nov;41(11):1284-92.

26. Cohen S, Kamarck T, Mermelstein R. A global measure of perceived stress. J Health Soc Behav. 1983 Dec;24(4):385-96.

27. Juárez SP, Merlo J. Revisiting the effect of maternal smoking during pregnancy on offspring birthweight: a quasi-experimental sibling analysis in Sweden. PLoS One. 2013 Apr 17;8(4):e61734. doi: 10.1371/journal. pone. 0061734
28. MacArthur C, Knox EG. Smoking in pregnancy: effects of stopping at different stages. Br J Obstet Gynaecol. 1988 Jun;95(6):551-5.

29. Dunkel Schetter C, Glynn LM. Stress in pregnancy: empirical evidence and theoretical issues to guide interdisciplinary research. In: Contrada RJ, Baum A, editors. The Handbook of stress science: biology, psychology, and health. New York: Springer; 2011. p. 321-43.

30. Roy-Matton N, Moutquin JM, Brown C, Carrier N, Bell L. The impact of perceived maternal stress and other psychosocial risk factors on pregnancy complications. J Obstet Gynaecol Can. 2011 Apr;33(4):344-52.

31. Hrubá D, Kachlík P. Influence of maternal active and passive smoking during pregnancy on birthweight in newborns. Cent Eur J Public Health. 2000 Nov;8(4):249-52.

32. Kukla L, Hrubá D, Tyrlík M; European Longitudinal Study of the Pregnancy and childhood. Smoking and damages of reproduction: evidence of ELSPAC. Cent Eur J Public Health. 2001 May;9(2):59-63.

Received August 9, 2013 Accepted in revised form March 18, 2014 